\title{
Unique Approach to Diagnosing and Treating Congenital Bronchial Atresia (CBA): A Case Series
}

Amit K Mahajan ${ }^{1 *}$, Rod Rahimi², Paul Vanderlaan², Erik Folch², Sidhu Gangadharan² and Adnan Majid ${ }^{2}$

${ }^{1}$ Department of Thoracic Surgery and Interventional Pulmonology, Inova Fairfax Hospital, 3300 Gallows Road, Falls church, Virginia, USA

${ }^{2}$ Division of Thoracic Surgery and Interventional Pulmonology, Beth Israel Deaconess Medical Center, Harvard Medical School, Boston, MA, USA

\begin{abstract}
Congenital bronchial atresia (CBA) is a rare airway anomaly resulting in termination of a bronchus into a blind pouch with associated mucoid impaction and segmental hyperinflation. The etiology of CBA is related to vascular insufficiency to the bronchial bud during embryologic development. Controversy exists regarding the best approach to treatment for $\mathrm{CBA}$ and relating symptoms to the presence of these anomalies can be challenging. This case series describes three patients with varying approaches to diagnosis and treatment of CBA.
\end{abstract}

Keywords: Bronchial atresia; Bronchoscopy

\section{Introduction}

Congenital bronchial atresia (CBA) is a rare anomaly of the pulmonary system. Isolated bronchial atresia can result in the termination of a bronchus into a blind pouch with associated mucoid impaction and hyperinflation of the obstructed lung segment [1-4]. The disorder is typically diagnosed in the second or third decade of life and has a male predominance, with an estimated prevalence of 1.2 cases per 100,00 males [5]. Bronchial atresia is believed to result from the insufficient supply of blood to the bronchial bud during embryologic development. [6,7]. Frequently, a mucocele will develop distal to the atretic airway due to recurrent infections. Additionally, collateral ventilation can result in emphysematous changes in surrounding lung that disturb normal respiratory mechanics.

The first case of bronchial atresia was reported in 1953, yet only approximately 100 cases have been documented since [4]. The left upper lobe is involved $64 \%$ of the time, most notably the apicoposterior segment. [8]. Hyperinflation associated with CBA stems from communications between normal pulmonary parenchyma through the pores of Kohn. Traditional presenting symptoms are observed in individuals who develop wheezing and recurrent pulmonary infections in the effected segment. Additionally, pneumothoraces have been associated with CBA [2]. Controversy exists regarding the best approach to the treatment of CBA. The general consensus appears to hold with conservative management in adults if infections are infrequent. Unfortunately if recurrent infections are present and medical treatment is ineffective, or malignant lesions cannot be excluded, surgical resection may be indicated.

This case series describes three patients with CBA presenting with shortness of breath and recurrent pulmonary infections over a 4 month period at a single institution. While radiographic findings were suggestive of bronchial atresia, bronchoscopy was used to identify the atretic airway and confirm the diagnosis in two of the cases. This communication will help adult pulmonologist become more knowledgeable of the spectrum of presenting symptoms, radiographic findings, and utility of flexible bronchoscopy in establishing the diagnosis of CBA.

\section{Case 1}

A 44-year-old female with a history of congenital lobar emphysema presented to the outpatient pulmonary clinic with shortness of breath on exertion. A hyperlucency over her left upper lung zone was discovered incidentally 15 years prior for workup of a possible rib fracture. The patient endorsed episodes of pneumonia every 2 years since childhood.
Each of these episodes required outpatient antibiotic therapy. She had been previously quite active, but noted new dyspnoea on exertion and difficulty ambulating up one flight of stairs.

The patient's past medical history and review of systems were negative. Her physical exam revealed distant breath sounds over the left upper chest, but was otherwise unremarkable. Pulmonary function testing was normal and revealed a forced expiratory volume in 1 second $\left(\mathrm{FEV}_{1}\right)$ of $93 \%$ of predicted, forced vital capacity (FVC) of $105 \%$ of predicted and $\mathrm{FEV}_{1} / \mathrm{FVC}$ ratio of 72 . The patients total lung capacity (TLC) was $111 \%$ of predicted, function residual capacity (FRC) was $126 \%$ of predicted, and her residual volume (RV) was $122 \%$ of predicted. The patient's diffusion capacity (DLCO) was $89 \%$ of predicted. The patient's chest X-ray revealed hyperlucency, extensive hyperinflation, and decreased vascularity in the left upper lung zone (Figure 1). The computer tomography (CT) scan of the chest revealed a hyperlucent apicoposterior segment of the left upper lobe and hypoplastic vasculature consistent with bronchial atresia (Figure 2). Her quantitative lung scan (Figure 3) reveals decreased perfusion to the upper third of the left lung, which corresponded to the region of hyperlucency seen chest X-ray and CT scan of the chest.

The patient was seen in the outpatient interventional pulmonology clinic for further investigation into and possible diagnosis and management of bronchial atresia. A pediatric flexible video bronchoscope (Olympus Model, Olympus America, BF-XP160F, $2.8 \mathrm{~mm}$ ) was maneuvered into the apicoposterior segment of the left upper lobe. After advancing to the fourth order airways, an airway was visualized with an abrupt termination ending in a blind pouch (Figure 4) consistent with the diagnosis of CBA. Considering her symptoms and findings on PFT, the patient was started on a trial of long and short acting bronchodilators prior to exercise. She was also scheduled for a full set of pulmonary function tests in 6 months. If symptoms continued to worsen and hyperinflation progressed surgical resection would be reconsidered.

${ }^{*}$ Corresponding author: Amit K Mahajan, Department of Thoracic Surgery and Interventional Pulmonology, Inova Fairfax Hospital, 3300 Gallows Road, Falls church, Virginia, USA, Tel: 7032805858; E-mail: bmahaj@gmail.com

Received April 03, 2017; Accepted April 20, 2017; Published April 24, 2017

Citation: Mahajan AK, Rahimi R, Vanderlaan P, Folch E, Gangadharan S, et al (2017) Unique Approach to Diagnosing and Treating Congenital Bronchial Atresia (CBA): A Case Series. J Pulm Respir Med 7: 402. doi: 10.4172/2161-105X.1000402

Copyright: (c) 2017 Mahajan AK, et al. This is an open-access article distributed under the terms of the Creative Commons Attribution License, which permits unrestricted use, distribution, and reproduction in any medium, provided the original author and source are credited. 


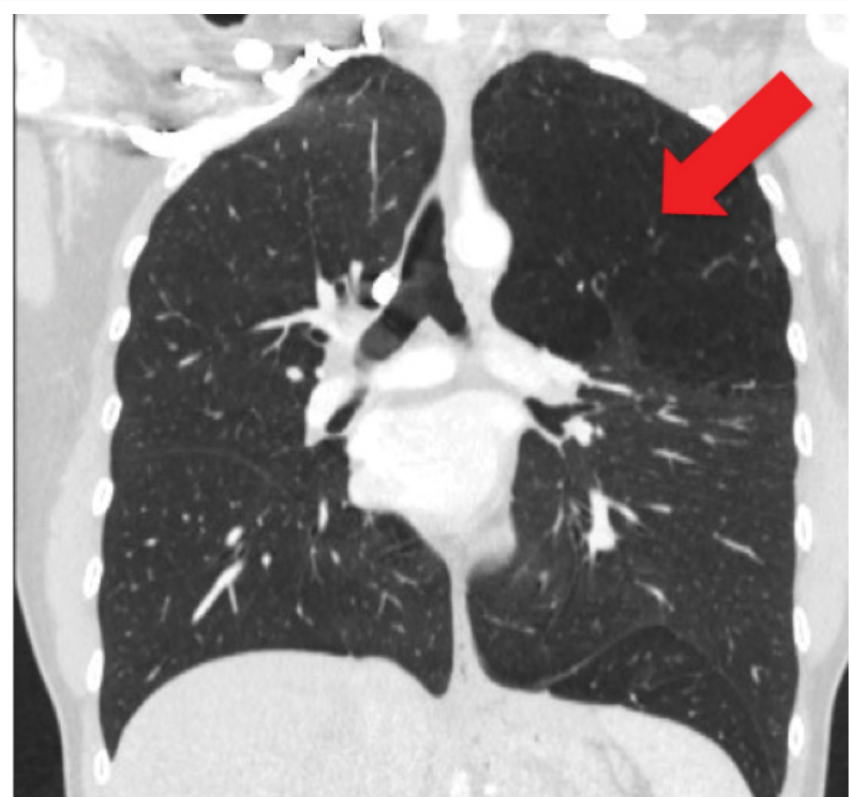

Figure 1: Chest X-ray revealed hyperlucency, extensive hyperinflation, and decreased vascularity in the left upper lung zone.

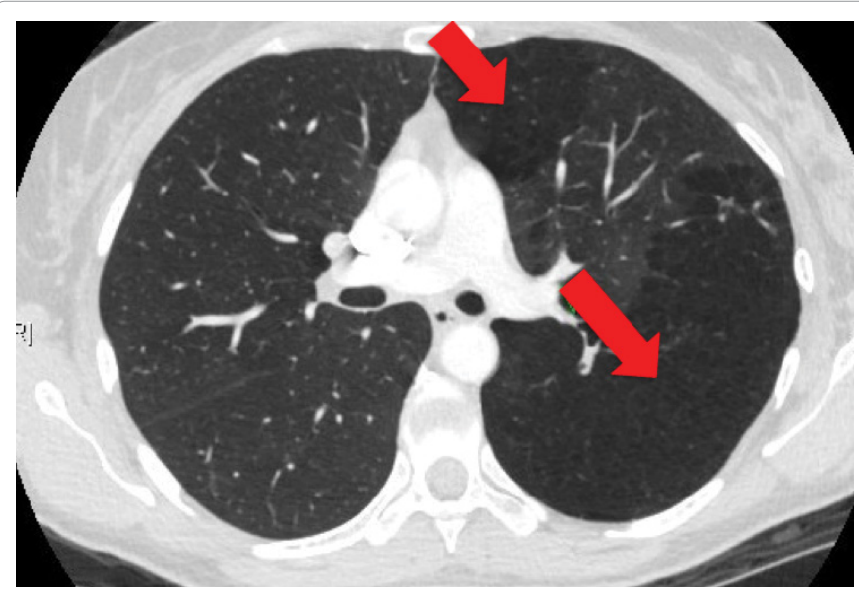

Figure 2: CT scan of the chest revealed a hyperlucent apicoposterior segmen of the left upper lobe and hypoplastic vasculature consistent with bronchial atresia.

\section{Case 2}

A 55-year-old male presented to the outpatient pulmonary clinic complaining of left sided chest pain. As part of his workup, the patient underwent a chest X-ray revealing a para-aortic lung parenchymal lucency. He described shortness of breath while walking up two flights of stairs, but did not have any other significant limitations. He endorsed episodes of bronchitis twice a year with a productive cough, which was treated as an outpatient with oral antibiotics.

The patient's past medical history was unremarkable and he did not take any medications. He drinks alcohol socially and briefly smoked cigarettes over thirty years ago. He did not engage in intravenous drug abuse. The review of symptoms was otherwise normal. His physical exam was normal with equal and appropriate breath sounds bilaterally. Pulmonary functions were consistent with mild obstruction, with an $\mathrm{FEV}_{1}$ of $87 \%$ of predicted, an $\mathrm{FVC}$ of $99 \%$ predicted, and an $\mathrm{FEV}_{1} / \mathrm{FVC}$ ratio of 67 . The patient did not have lung volumes or diffusion capacity

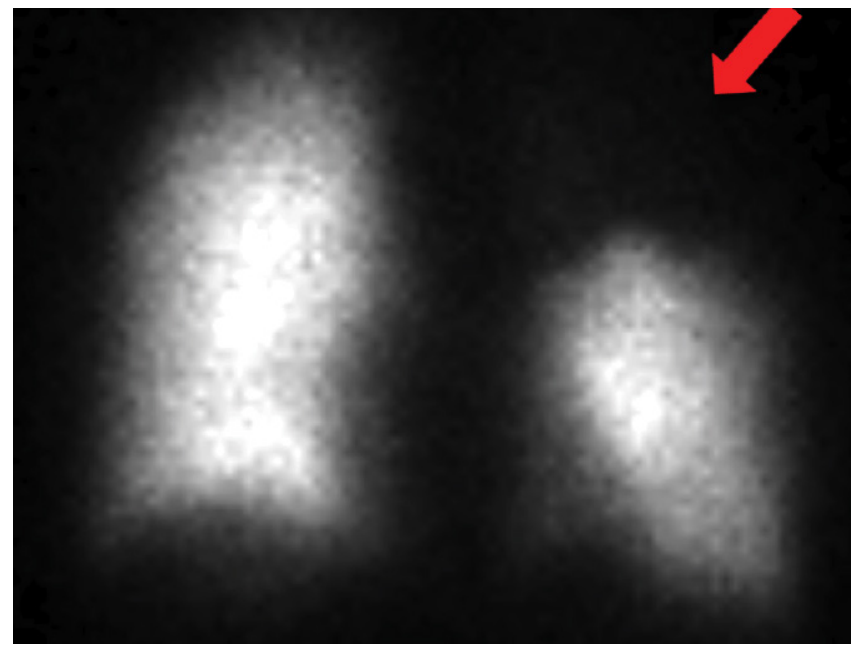

Figure 3: Lung scan.

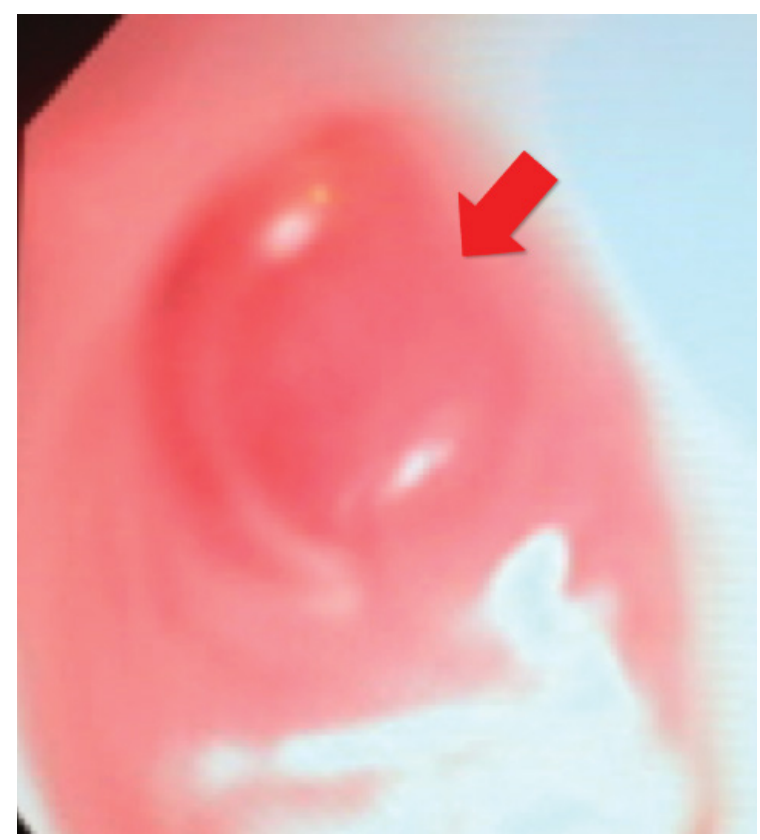

Figure 4: Airway was visualized with an abrupt termination ending in a blind pouch in interventional pulmonology.

at this time. The patient's chest CT scan revealed a possible atretic lingular bronchus with layering secretions (Figure 5).

The patient was seen in the Interventional Pulmonary clinic for further investigation of his lingular abnormality. The patient underwent flexible bronchoscopy to better characterize the bronchial abnormality and confirm if the patient had an atresia or a web. The pediatric bronchoscope (Olympus Model, Olympus America, BF-XP160F, 2.8 $\mathrm{mm}$ ) was used to enter the inferior segment of the lingula where a blind pouch was identified at the site of an atretic airway (Figure 6). Saline was instilled in the airway without signs of airway patency. A trial of bronchodilators was recommended with follow-up in the Interventional Pulmonary clinic in 2 months. If medical management failed and the patient's symptoms persisted on exertion, surgical resection would be considered. 


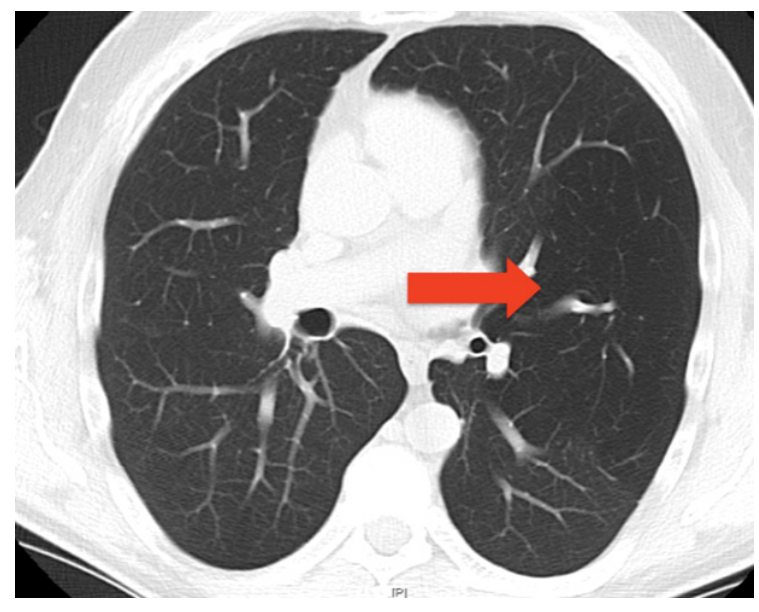

Figure 5: Chest CT scan revealed a possible atretic lingular bronchus with layering secretions.

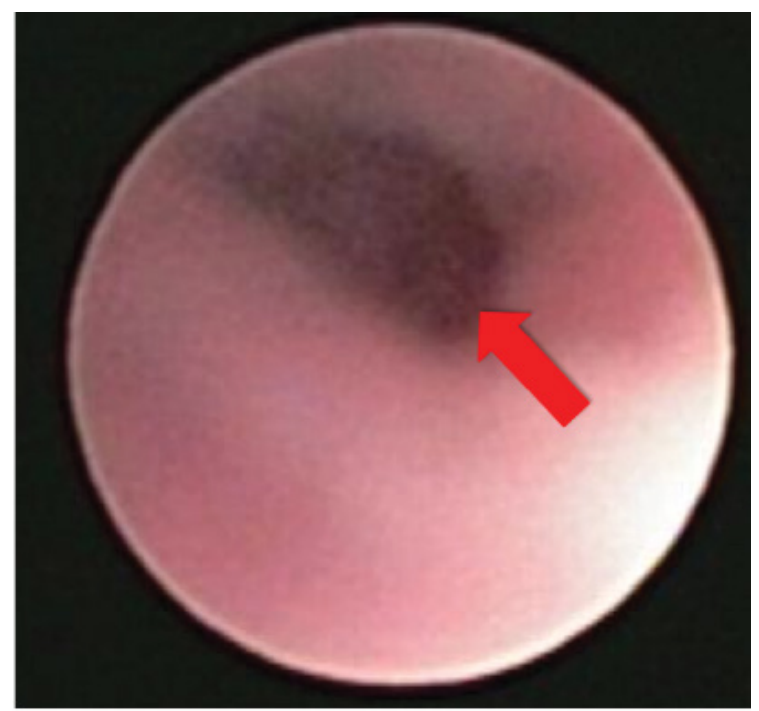

Figure 6: Pediatric bronchoscope showing the inferior segment of the lingula where a blind pouch was identified at the site of an atretic airway.

\section{Case 3}

A 21-year-old female presented to outpatient thoracic surgery clinic with recurrent left upper lobe lung infections. She was treated with antibiotics for five episodes of pneumonia from October 2012 to October 2013. The patient endorsed intermittent left upper back pain, occasional substernal chest pain, fatigue, and a chronic, productive cough. She did not describe fevers, chills, or night sweats. A CT scan of the chest revealed left upper lobe bronchiectasis likely related to recurrent infections and the presence of an atretic bronchus in the apicoposterior segment of the left upper lobe (Figure 7). A flexible bronchoscopy was performed without visualization of a blind pouch in the left upper lobe. A bronchoalveolar lavage (BAL) was performed that grew Pseudomonas aeruginosa on culture, which was subsequently treated.

The patient's past medical history was unremarkable. She was a never smoker, did not drink, and has never engaged in intravenous drug

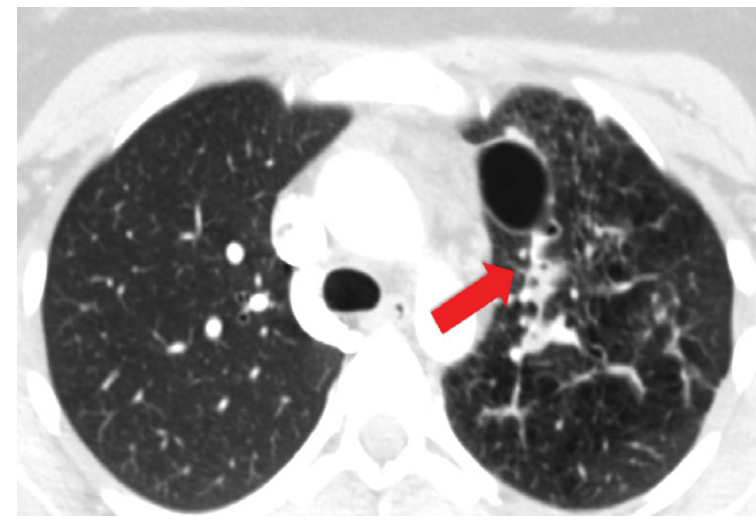

Figure 7: CT scan of the chest revealed left upper lobe bronchiectasis.

abuse. Her review of symptoms was otherwise normal. Her physical exam was normal with equal and appropriate breath sounds bilaterally.

Pulmonary functions were performed revealing an $\mathrm{FEV}_{1}$ of $91 \%$ of predicted, an FVC of $98 \%$ predicted, an $\mathrm{FEV}_{1} / \mathrm{FVC}$ ratio of 80 , and a DLCO $77 \%$ of predicted. The CT scan report described a likely atretic left upper lobe apicoposterior segment with associated pneumatocele and emphysematous changes.

The patient was seen in the thoracic surgery clinic for possible left upper lobe apicoposterior segmentectomy. Considering the patient's recurrent episodes of left upper lobe pneumonia and radiographic evidence of bronchiectasis, the patient underwent video assisted thoracoscopic (VATS) surgical resection of the upper lobe apicoposterior segment. The patient tolerated the procedure well and was discharged three days after surgery. Histologic review of the resected segment revealed bronchiectatic lung with scarring and emphysematous changes. Two weeks following surgery, the patient did not complain of any respiratory symptoms and her chest X-ray was normal with expected post-operative changes. Three months after surgery, the patient presented to the outpatient clinic with back to her pre-surgical baseline and had no further respiratory infections since resection.

\section{Discussion}

Although CBA is a congenital bronchopulmonary malformation that can be recognized in childhood, it is often identified in adulthood with presentations ranging from incidental radiographic abnormality to recurrent pneumonia [1]. While approximately 100 cases of CBA have been reported in the literature, the true incidence of this entity is unknown, as many individuals are asymptomatic and never seek medical attention. In fact, sixty percent of patients with CBA are asymptomatic and their anomaly is only discovered on a routine chest radiograph [3]. Awareness of these anomalies is necessary for pulmonologists as identification of these abnormalities is typically in the second and third decades of life.

Atretic bronchi lack direct communication with the central bronchial tree. Hyperinflation is typically present as a result of collateral ventilation through the bronchoalveolar channels of Lambert, pores of Kohn, or interbronchiolar channels. Since the bronchial tree distal to the atresia has been found to branch normally, [9] bronchial secretions can be produced and accumulate distal to the atretic airway and result in recurrent infections. In patients with a history of recurrent pneumonia without clear etiology, further radiographic imaging and bronchoscopic 
investigations are warranted. Hyperlucency discovered on chest X-ray or CT scan, particularly affecting the left upper lobe, should raise concern for CBA. While high resolution CT scan offers a sensitive imaging modality to identify lesion in the lung parenchyma, the tool is less reliable when identifying airway pathology. In cases of presumed bronchial atresia, bronchoscopy with identification of the offending obstructed airway is invaluable. Visualization of the pathologic airway can differentiate between focal airway stenosis, bronchial webs or endobronchial tumours that can be intervened upon bronchoscopically, or confirm the presence of airway atresia. A high resolution CT chest revealed evidence of an atretic bronchus and bronchoscopy was used to confirm the diagnosis in two cases described in this case series. This supports previous reports demonstrating that in symptomatic patients with evidence of lobar hyperlucency, high resolution CT of the chest is a valuable modality for identifying bronchial atresia, but evaluation with flexible bronchoscopy is also necessary [10].

Dyspnoea related to the presence of CBA likely results from the obstructive pulmonary defect and hyperinflation from collateral ventilation between normal and abnormal lung. Hyperinflated lung creates a mechanical disadvantage of the ipsilateral diaphragm due to resultant flattening of the muscle with subsequent poor contraction. Inadequate contraction the diaphragm precludes adequate force to generate the appropriate negative pressure necessary for inspiration. Such a mechanical disadvantage can result in sensation of dyspnoea and increased work of breathing as other respiratory muscles must carry the burden of the poorly functioning diaphragm. All of the patients featured in this case series suffered from dyspnoea on exertion in addition to recurrent pneumonia ranging in time periods from yearly to monthly. As seen in our patients, these symptoms are often manifested during exertion, which may be related to some degree of dynamic hyperinflation.

The management of CBA remains controversial. The main indication for surgical intervention includes recurrent infections and significant shortness of breath. After identification of the atretic airways, the patients in the first two cases were prescribed short and long acting bronchodilators, with the goal to decrease airflow obstruction and air trapping and plan to reassess symptoms and pulmonary function tests after three months. The effectiveness of bronchodilators in patients with CBA and presumed normal airway hyperresponsiveness is unknown. However, given the low risk of administering bronchodilators it is a reasonable starting intervention. In regards to recurrent infections and bronchiectasis in patients with CBA, there is no consensus regarding the point when the benefits of surgical resection outweigh the risks. Yet, in cases of recurrent infection with associated bronchiectasis in patients with CBA, the affected lung is unlikely to be contributing to meaningful ventilation. In this case, resection resulting in improved diaphragmatic excursion and reduction in V/Q mismatch is recommended. Moving forward, systematic and prospective data collection of various treatments of CBA should be performed to evaluate responses to conservative and surgical management.

While CBA remains a rare disorder, the increased utilization of screening CT scans will continue to identify asymptomatic patients with this pathology. Pulmonologist should recognize the possible symptoms associated with this disorder and feel comfortable counselling those patients. While radiographic findings are essential for identifying patients who may have CBA, bronchoscopic confirmation should always be attempted to rule out other possible etiologies for segmental hyperinflation. While there is no consensus regarding the therapies for minimally symptomatic patients, patients with recurrent infections and associated bronchiectasis are regularly referred for surgical intervention. As this disorder is further understood, the outcomes and success rates of the various therapeutic options will become clearer.

\section{References}

1. Barreiro TJ, Gemmel DJ (2009) A 21-year-old woman with dyspnea and an abnormal chest radiograph. Chest 135: 550-553.

2. Berkman N, Bar-Ziv J, Breuer R (1996) Recurrent spontaneous pneumothorax associated with bronchial atresia. Respir Med 90: 307-309.

3. Jederlinic PJ, Sicilian LS, Baigelman W, Gaensler EA (1987) Congenital bronchial atresia. A report of 4 cases and a review of the literature. Medicine (Baltimore) 66: 73-83.

4. Ramsay BH, Byron FX (1953) Mucocele, congenital bronchiectasis, and bronchiogenic cyst. J Thorac Surg 26: 21-30.

5. Pasthakis K, Lachanis S, Kotoulas C, Koutoulidis V, Panagou P, et al. (2004) The prevalence of congenital bronchial atresia in males. Monaldi Arch Chest Dis 61: 28-34.

6. Williams AJ, Schuster SR (1985) Bronchial atresia associated with bronchogenic cyst. Evidence of early appearance of atretic segments. Chest 87: 396-398.

7. Zylak CJ, Eyler WR, Spizarny DL, Stone CH (2002) Developmental lung anomalies in the adult: radiologic-pathologic correlation. Radiographics 22: S25-S43.

8. Kinsella D, Sissons G, Williams MP (1992) The radiological imaging of bronchial atresia. Br J Radiol 65: 681-685.

9. Schuster SR, Harris GB, Williams A, Kirkpatrick J, Reid L (1978) Bronchial atresia: a recognizable entity in the pediatric age group. J Pediatr Surg 13: 682-689.

10. Ward S, Morcos SK (1999) Congenital bronchial atresia-Presentation of three cases and a pictorial review. Clin Radiol 54: 144. 